\title{
Risk of COVID-19 infection in patients with rheumatic disease taking disease-modifying anti-rheumatic drugs
}

\author{
Behnaz Yousefghahari ${ }^{1}$ (1) Sanaz Navari ${ }^{2} \cdot$ Mahmoud Sadeghi $^{1} \cdot$ Shima Soleimaniamiri ${ }^{3}$. \\ Mohammadjafar Soleimaniamiri $^{3} \cdot$ Behzad Heidari $^{1} \cdot$ Mansour Babaei $^{1} \cdot$ Kian Ghodrati $^{4} \cdot$ Ardeshir Guran $^{5}$. \\ Hemmat Gholinia ${ }^{1}$
}

Received: 18 April 2021 / Revised: 15 May 2021 / Accepted: 19 May 2021 / Published online: 29 May 2021

(c) International League of Associations for Rheumatology (ILAR) 2021

\begin{abstract} individuals without RD. after adjusting for all covariates using logistic regression analysis. and that HCQ therapy has no influence on the risk of COVID-19 infection.

Key points

- The risk of COVID-19 infection is not higher in patients with RD

on DMARD therapy.

- The prevalence of COVID-19 infection in HCQ users has not

significant difference relative to non-users.

- Significant percent of RD patients taking DMARDs had asympto-

matic infection.

- There was a positive association between leflunamide therapy and the risk of COVID-19 infection.
\end{abstract}

Background and objective Patients with rheumatic disease taking long-term disease-modifying anti-rheumatic drugs (DMARDs) are expected to have a higher risk of infection due to the alterations in cellular immunity associated with these medications. However, the potential risks associated with these drugs remain unclear. This study aimed to estimate the risk of COVID-19 infection in patients with rheumatic disease taking disease-modifying anti-rheumatic drugs.

Methods Patients with autoimmune rheumatic disease taking DMARDs with or without long-term ( $>6$ months) HCQ treatment prior to the COVID-19 outbreak were selected consecutively. The diagnosis of COVID-19 was made based on the history of symptoms suggestive of the disease and/or serum IgG positivity. During statistical analysis, the risk of COVID-19 infection was calculated in rheumatic patients taking DMARDs versus controls, as well as in patients taking HCQ versus those who are not. The ORs and $95 \%$ CIs were also calculated. The participants in the control group were selected from

Results A total of 800 patients with RD and 449 controls were analyzed. COVID-19 infection was detected in $16.8 \%$ of rheumatic patients versus $17.6 \%$ of controls (OR 0.95; 95\% CI 0.7-1.28). The proportions of COVID-19 infection in HCQ users versus non-users were $15.3 \%$ and $18.1 \%$, respectively (OR 0.87; 95\% CI 0.61-1.26). These results remained unchanged

Conclusion These findings indicate that rheumatic patients taking DMARDs are not at a higher risk of COVID-19 infection,

Behnaz Yousefghahari

behnaz_ghahari@yahoo.com

1 Clinical Research Development Unit of Rohani Hospital, Babol University of Medical Sciences, Ganjafrooz Ave, Babol, Iran

2 Babol University of Medical Sciences, Babol, Iran

3 Razi Labratory, Babol, Iran

4 Wilfrid Laurier University, Waterloo, Canada

5 Institute of Structronics, Ottawa, Canada 
Keywords COVID-19 - Disease-modifying anti-rheumatic drugs $\cdot$ Hydroxychloroquine $\cdot$ Infection risk $\cdot$ Rheumatic patients

\section{Introduction}

Since the initial outbreak in China, the coronavirus disease (COVID-19) has continued to spread and remains an important cause of mortality across numerous geographic regions (1). Despite the development of several vaccines to prevent transmission, COVID-19 transmission remains a problem as it may still be possible for an individual to spread the virus after being vaccinated. Therefore, until these vaccines are able to prevent secondary infections, the use of masks, observation of quarantine practices, and social isolation are effective and important strategies for controlling the spread of the disease (2-4).

The first generation of COVID-19 vaccines is expected to reduce the transmission of severe acute respiratory syndrome coronavirus 2 (SARS-CoV-2). However, suppression of community transmission requires immunity in $20-50 \%$ of the population. Although these vaccines may confer protective effects against COVID-19 infection, their influence on infection rate, and thus transmission rate, remains to be seen (5).

Given the worldwide spread of COVID-19, observing quarantine practices, enforcing social distancing, identification of high-risk populations, and implementing pharmacologic interventions to stop the spread of infection in the general population should be considered (2-4).

Several factors have been associated with an increased risk of COVID-19 development, as well as increased transmission and fatality. A systematic review and meta-analysis found that hypertension, cardiovascular disease, history of smoking, and diabetes were present in $16.37 \%, 12.11 \%$, $7.63 \%$, and $7.87 \%$ of patients infected with COVID-19, respectively (6). Infection occurs more frequently in middleaged and older individuals, particularly those with chronic comorbidities such as cardiovascular disease, hypertension, and diabetes, as well as chronic lung, renal, and liver diseases (7). Rheumatic patients, such as those with rheumatoid arthritis (RA) and systemic lupus erythematosus, who are taking disease-modifying anti-rheumatic drugs (DMARDs) are at a higher risk of infection and have greater morbidity and mortality than the general population. These effects may be due to the medications themselves or from alterations in cellular immunity (8-10).

However, this issue has not been adequately investigated, and the potential risks conferred by treatment with DMARDs remain unclear. While methotrexate therapy itself decreases disease activity, it may also increase the risk for infections (11).
Data from the COVID-19 Global Rheumatology Alliance physician-reported registry demonstrated that in patients with rheumatic disease, exposure to prednisolone at a dose of $>10 \mathrm{mg} /$ day was associated with higher odds of hospitalization, while the use of anti-TNF was associated with reduced odds of hospitalization (12).

A review of 69 studies that summarized the advantages and disadvantages of current immunosuppressive drugs on COVID-19 infection found contradictory results (13). This may be partly attributed to the different mechanisms of action of these drugs on the immune system or to variations in the combinations of DMARDs used in the treatment of rheumatic disease. As a result, while a number of DMARDs may increase the probability of acquiring COVID-19 infection, antimalarial drugs that are widely used in rheumatic patients may provide potential benefits against COVID-19 infection (14).

Antimalarial drugs have demonstrated antiviral activity through their PH-dependent effects on several viruses, including coronaviruses. A systematic review of 6 studies and 23 ongoing clinical trials showed that chloroquine could effectively limit the replication of SARS-CoV-2 in vitro (6). Therefore, in patients with rheumatic disease, the administration of hydroxychloroquine (HCQ), irrespective of its immunomodulatory effects, might decrease the risk of COVID-19 infection. For these reasons, the safety of the use of immunosuppressive drugs during the COVID19 pandemics remains questionable, and the prevalence of COVID-19 infection in rheumatic patients varies across different studies.

Nonetheless, current data do not provide overwhelming evidence that patients with rheumatic disease, when compared with other comorbidities, are at a higher risk of COVID-19 infection. In one study of Portuguese patients with autoimmune diseases, treatment with HCQ was associated with a lower prevalence of COVID-19 infection (15). However, data regarding the distribution of COVID-19 infection in rheumatic patients are scarce, and the risk of COVID-19 infection in patients taking certain immunosuppressive drugs is particularly unclear. Therefore, we conducted the present study to determine the risk of COVID-19 infection in rheumatic patients who are using DMARDs versus the general population, and to assess the risk of COVID19 infection in rheumatic patients who use HCQ versus those who do not.

\section{Patients and methods}

This observational study was composed of 800 patients with inflammatory rheumatic disease aged 18 years and older who presented for follow-up clinical examination in a rheumatology clinic between May 1, 2020, and November 2020. 
At the time of study, each patient was taking one or more DMARDs, such as methotrexate, HCQ, low-dose prednisolone, anti-TNF drugs, or other immunosuppressive medications, for more than 6 months (between 8 and 110 months). See Table 1.

The diagnosis of COVID-19 infection was made based on clinical symptoms in addition with combination of positive laboratory tests, such as PCR or antibodies, and/or CT findings. The participants of the control group were selected from asymptomatic individuals who presented to the same laboratory for COVID-19 testing prior to elective surgery during the study period. The subjects in the control group had no current or history of rheumatic diseases and were not taking anti-rheumatic drugs. Serum $\mathrm{IgG}$ was measured by ELISA according to the manufacturer's instructions using an IgG antibody kit against SARS-Cov-2 provided by Pishtaz Teb, Tehran, Iran.

The manufacturer-reported sensitivity and specificity of the ELISA kits were $94.1 \%$ and $98.3 \%$, respectively (17). IgG antibody serum values $>1.1 \mathrm{AU} / \mathrm{mL}$ were considered positive. All tests were performed at the Razi Medical Diagnostic Laboratory in Babol, Iran.

Individuals who (1) had chronic allergic symptoms, (2) had irregular follow-up consults, and (3) were non-adherent to medications were excluded from the study.

Table 1 Characteristics of the study population with rheumatic diseases

\begin{tabular}{ll}
\hline Variables & $N(\%)$ \\
\hline Age $>50$ years & $479(59.5)$ \\
Obesity $\left(\mathrm{BMI} \geq 30 \mathrm{~kg} / \mathrm{m}^{2}\right)$ & $206(25.8)$ \\
Diabetes & $94(11.8)$ \\
Hypertension & $56(7)$ \\
History of cardiovascular disease & $30(3.8)$ \\
History of respiratory disease & $58(7.3)$ \\
Rheumatic diseases & \\
$\quad$ Rheumatoid arthritis & $473(59.1)$ \\
$\quad$ Systemic lupus erythematosus & $110(13.8)$ \\
$\quad$ Other diseases (PSA, SS, PM,DM, SCL) & $217(27.1)$ \\
Medications & \\
$\quad$ Hydroxychloroquine & $430(46.3)$ \\
$\quad$ Methotrexate & $467(58.4)$ \\
$\quad$ Prednisolone & $716(89.4)$ \\
Leflunomide & $176(22.4)$ \\
Anti-TNF drugs & $81(10.1)$ \\
Other medications & $59(7.4)$ \\
Duration of treatment with DMARDs, months, median & $24(8-110)$ \\
(range) & \\
\hline
\end{tabular}

$P S A$ psoriatic arthritis, $S S$ Sjogren syndrome, $P M$ polymyositis, $D M$ dermatomysitis, $S C L$ scleroderma,

$D M A R D s$ disease-modifying anti-rheumatic drugs
All participants were evaluated for history of COVID-19 infection or contact with a known case. Data were provided through face-to-face interviews in accordance with a questionnaire made to assess pre-specified signs and symptoms suggestive of COVID-19 infection, such as the presence of cough, shortness of breath, difficulty breathing, fever, chills, rigors, myalgia, headache, sore throat, and new olfactory or taste disorders. In addition, data were collected with respect to household or occupational contacts, especially contact with confirmed cases of COVID-19 infection which were defined as either hospitalization or a positive PCR test for the virus. Symptomatic disease was defined as the presence of any above symptoms, in addition to positive PCR or IgG $\mathrm{Ab}$ or CT scan confirming COVID-19. However, asymptomatic disease was defined as positive $\mathrm{PCR}$ or IgG Ab against COVID-19 without any of the abovementioned symptoms.

In the statistical analysis, the proportions of COVID-19 infection between rheumatic patients and the controls were compared. Furthermore, the proportion of COVID-19 infection was compared between HCQ users and non-users, as well as between HCQ users and the control group. The $\chi^{2}$ test, with calculation of the ORs and 95\% CIs, was used to determine the risk of COVID-19 infection.

Multiple logistic regression analysis was applied to examine the independent association between COVID-19 infection and HCQ treatment; COVID-19 infection was treated as the dependent variable while other variables, such as medications and demographic features, were treated as covariates. All analyses were performed using SPSS software version 18. This study protocol was approved by the Ethics Committee of Babol University of Medical Sciences, Babol, Iran (code IR.MUBABOL.HRI.REC.1399.344).

\section{Results}

A total of 800 patients with rheumatic disease and 449 controls were analyzed. One hundred thirty-five out of the 800 rheumatic patients (16.8\%) and 79 out of the 449 controls (17.6\%) had COVID-19 infection (OR 0.95; 95\% CI 0.7-1.28). The characteristics of study patients are shown in Tables 1 and 2.

Seventy-three $(54 \%)$ of 135 rheumatic patients with COVID-19 infection were symptomatic and the rest (46\%) had asymptomatic infection. All patients in the control group were asymptomatic. Thirty-one out of 135 COVID19 infections in the patient group (23\%) were hospitalized for a median duration of 6 (range 3-12) days and there was no mortality.

In the statistical analysis, the two groups of symptomatic and asymptomatic rheumatic patients did not show significant differences in age, disease duration, and distribution of DMARDs (data are not shown). 
Table 2 Distribution of demographic features and self-reported symptoms in 73 patients with symptomatic COVID-19 infection

\begin{tabular}{ll}
\hline Demographic features or symptoms & $n(\%)$ \\
\hline Age $>50$ years & $45(61.6)$ \\
Fever and chills & $57(78.1)$ \\
Myalgia & $15(20.5)$ \\
Gastrointestinal symptoms & $25(34.2)$ \\
Headache & $12(16.4)$ \\
Olfactory or taste disorders & $20(27.4)$ \\
Respiratory symptoms & $58(79.2)$ \\
Obesity (BMI $\left.\geq 30 \mathrm{~kg} / \mathrm{m}^{2}\right)$ & $30(41.7)$ \\
PCR positivity & $12(16.4)$ \\
Serum IgG positivity & $3(4.1)$ \\
Increased C-reactive protein or ESR & $3(4.1)$ \\
Compatible findings in lung CT scan & $41(56)$ \\
Number of hospitalizations & $31(23)$ \\
Family contact with a known case & $44(60.2)$ \\
Diabetes & $13(18.1)$ \\
Hypertension & $9(12.5)$ \\
Cardiovascular diseases & $2(2.8)$ \\
\hline
\end{tabular}

The prevalence of COVID-19 infection in HCQ users and non-users were $15.3 \%$ and $18.1 \%$ (Table 3 ), respectively (OR $0.87,95 \%$ CI 0.61-1.26). When compared with controls, the odds of COVID-19 infection in HCQ users was 0.9 (95\% CI 0.63-1.28). The risk of COVID-19 infection in HCQ users versus non-users, after adjustment for all covariates such as DMARDs other than HCQ and demographic factors, are shown in Table 4. After adjustment for all covariates, multiple logistic regression analysis showed that the risk of COVID-19 infection in HCQ users remained non-significant, suggesting that there is no association between the risk of COVID-19 infection and use of other DMARDs including methotrexate, low-dose prednisolone, and anti-TNF drugs except leflunomide. There was a positive association between leflunomide therapy and the risk of COVID-19 infection (OR 1.95; 95\% CI 1.08-3.5).

Furthermore, close contact with confirmed COVID-19 cases in the family was independently associated with an increased risk of COVID-19 infection (adjusted OR 38.6; 95\% CI 14.39-103.8) (Table 4). Subgroup analyses of patients with RA and non-RA diseases did not change the risk of COVID-19 infection.

Table 3 Distribution of COVID-19 infection in rheumatic diseases treated with and without hydroxychloroquine

\begin{tabular}{llll}
\hline Variable & $\begin{array}{l}\text { Hydroxychloro- } \\
\text { quine }-(n=370)\end{array}$ & $\begin{array}{l}\text { Hydroxychloro- } \\
\text { quine }+(\mathrm{n}=430)\end{array}$ & $P$ value \\
\hline $\begin{array}{c}\text { COVID-19 infec- } 67(18.1 \%) \\
\text { tion }(n=135)\end{array}$ & $68(15.3)$ & 0.27 \\
\hline
\end{tabular}

Table 4 Association between pre-exposure ${ }^{\mathrm{a}}$ hydroxychloroquine (HCQ) treatment and acquisition of COVID-19 in rheumatic patients taking disease-modifying anti-rheumatic drugs

\begin{tabular}{lrc}
\hline Predictors & OR $(95 \%$ CI $)$ & Adjusted OR (95\% CI) \\
\hline HCQ & $0.89(0.62-1.28)$ & $0.76(0.41-1.38)$ \\
Prednisolone & $0.75(0.43-1.31)$ & $0.76(0.23-2.5)$ \\
Methotrexate & $1.01(0.69-1.46)$ & $1.16(0.56-2.41)$ \\
Anti-TNF drugs & $63(32-1.27)$ & $0.58(0.18-1.84)$ \\
Leflunomide & $1.93(1.29-2.89)$ & $1.95(1.08-3.5)$ \\
Other drugs & $72(33-1.56)$ & $0.64(0.079-5.22)$ \\
Age $>50$ years & $1.30(89-1.90)$ & $0.86(0.43-1.74)$ \\
Obesity (BMI $\geq 30 \mathrm{~kg} /$ & $1.74(1.17-2.57)$ & $1.11(0.60-2.05)$ \\
$\left.\quad \mathrm{m}^{2}\right)$ & & \\
Diabetes & $1.43(0.84-2.41)$ & $1.17(0.54-2.54)$ \\
Hypertension & $1.17(59-2.33)$ & $0.72(0.24-2.14)$ \\
Cardiovascular disease & $1.46(0.61-3.49)$ & $1.82(0.57-5.87)$ \\
Household contact & $41.6(19.07-90.01)$ & $38.6(14.39-103.8)$ \\
$\quad$ with a known & & \\
COVID-19 case & & \\
\hline
\end{tabular}

The association was determined using multiple logistic regression analysis with calculation of OR and 95\% CI after adjustment for all covariates including drugs, demographic and epidemiological factors

${ }^{\text {a } I n i t i a t i o n ~ o f ~ t r e a t m e n t ~ s e v e r a l ~ m o n t h s ~ p r i o r ~ t o ~ C O V I D-19 ~ o u t b r e a k ~}$

\section{Discussion}

The findings of the present study indicate that patients with $\mathrm{RD}$ on long-term DMARD therapy were not at a higher risk of COVID-19 infection than the general population. Furthermore, HCQ therapy had no influence on the risk of COVID19 infection. Although the prevalence of COVID-19 infection in HCQ users was lower than that in non-users (15.3\% and $18.1 \%$, respectively), the difference was not statistically significant. Individual analysis of patients with RA and nonRA diseases did not change the results.

The findings of this study regarding COVID-19 infection are consistent with the results of a recent large population-based cross-sectional study. In that study, SARSCOV-2 antibody positivity was detected in $17.1 \%$ (95\% CI $14.6-19.5 \%)$ of randomly selected participants from the general population of Iran (17). In another study, the prevalence of COVID-19 infection in the general population of different countries varied from $<0.1$ to $>20 \%$ (18).

The lack of association between DMARD therapy and the risk of COVID-19 infection has also been shown in a cross-sectional study of 10,260 rheumatic patients from Italy; treatment with DMARDs and other small molecules were shown to have no influence on the susceptibility to COVID-19 infection when compared with the general population (19).

Interestingly, $46 \%$ of COVID-19 infections in rheumatic patients of the present study were asymptomatic, indicating 
that a significant proportion of patients taking DMARDs may acquire asymptomatic COVID-19 infection. This may be partly dependent on the overlap of symptoms of COVID19 and those of rheumatic diseases. Thus, in a proportion of patients with COVID-19, the occurrence of mild symptoms may be attributed to the exacerbation of underlying rheumatic diseases.

In contrast, two studies found a higher rate of COVID-19 infection in rheumatic patients than in those without rheumatic diseases $(8,9)$. In a multicenter retrospective study of 42 families with a documented history of COVID-19 exposure, rheumatic patients had a higher susceptibility to COVID-19 infection compared to those without RD. In this study, COVID-19 infection developed in 27 of $43(63 \%)$ patients with RD versus 28 out of $83(34 \%)$ patients without RD (OR 2.68; 95\% CI 1.14-6.27). However, these findings are limited as the data were collected via telephone calls and not through direct interviews, as was done in our study (8). Similarly, Bozzella et al. (9) found a higher rate of COVID19 infection in SLE patients who were on long-term HCQ for more than 6 months than in the general population $(2.5 \%$ vs. $0.76 \%$, respectively). However, the course of COVID-19 in SLE patients was mild and self-limiting (9), which is consistent with the high prevalence of asymptomatic patients in our study. In this study, the reason for the increased risk of COVID-infection with leflunomide therapy is unclear, and data in this context are scarce. In a separate smaller study, leflunomide therapy resulted in shorter viral shedding time in 5 out of 10 patients (20). However, in another study with a larger sample size, treatment with leflunomide had no influence on viral clearance or duration of hospital stay (21).

The impact of pre- or post-exposure prophylaxis using HCQ on the risk of COVID-19 infection, which has been addressed in several studies, yielded conflicting results in patients without rheumatic disease (22-28). The results of three randomized clinical trials and two observational studies were similar to those of our study. In these studies, pre-exposure prophylaxis with HCQ did not affect the risk of COVID-19 infection (22-26). Similarly, two RCTs that examined the effect of post-exposure prophylaxis with HCQ found no significant decrease in the risk of COVID-19 infection $(27,28)$. Nonetheless, a few studies have shown that HCQ has a preventive effect against SARS-CoV-2 infection $(15,30-32)$. Contradictory findings can be explained by several factors, including study design, diagnostic method for COVID-19 detection, method of data collection, and the time of HCQ administration before or after exposure. Administration of HCQ after or prior to exposure may not provide the required serum concentrations of the drug to inhibit SARS-Cov-2. As a result, the effect of HCQ is delayed until the serum concentration reaches therapeutic levels. In contrast, rheumatic patients receiving long-term HCQ therapy achieve a steady state over time and are more likely to attain adequate serum levels at the time of exposure. However, a previous study found that the mean serum and plasma levels of HCQ in rheumatic patients taking long-term HCQ were almost one tenth of the concentration required for in vitro viral inhibition (33). Therefore, the lack of a significant difference between HCQ users and non-users in this study may be attributed to the insufficient serum concentrations of HCQ. Another limitation of our study is the small sample size; it may be possible to detect a significant difference if a larger sample size is used.

One of the strengths of our study is the consecutive selection of rheumatic patients who were taking long-term DMARDs with or without HCQ. The diagnosis of patients and controls was confirmed by serum IgG positivity. In areas with a high prevalence of infection, elevated levels of serum $\mathrm{IgG}$ are suggestive of previous exposure to or current infection with SARS-Cov-2 (34). Thus, this test is helpful for identifying people who were previously infected with COVID-19. A meta-analysis revealed that IgG antibodies have a sensitivity and specificity of $97 \%$ and $98 \%$, respectively, for diagnosing COVID-19 (35). Because the patients and the control group were selected simultaneously, the risk of exposure to COVID-19 infection was similar between the two.

\section{Conclusion}

In conclusion, the results of this study indicate that rheumatic patients taking long-term immunosuppressive medications are not at a higher risk of COVID-19 infection. Furthermore, chronic exposure to HCQ in rheumatic patients does not significantly reduce the risk of COVID-19 infection. This study adds new information to the existing data and suggests that further studies with larger sample sizes are needed to clarify the impacts of DMARDs, particularly HCQ, on the risk of COVID-19 infection. There is still a long way to go before demonstrating the long-term protective effects of vaccination.

Acknowledgements We would like to thank the clinical research development unit of Rohani Hospital of Babol and Razi Laboratory for providing funds to perform this research.

Author contributions B.Y.G.H., B.H., M.B., S.N., M.S., A.G., K.G.H., M.J.S., and S.H.S. conceived the research question, designed the protocol, and were involved in the literature search, study selection, and data extraction. B.H., B.Y.G.H., M.S., and M.B. contributed to data acquisition, analysis, and interpretation. B.Y.G.H., B.H., M.B., S.N., M.S., A.G., K.G.H., M.J.S., and S.H.S. created the tables; and B.Y.G.H., B.H., M.B., S.N., M.S., A.G., K.G.H., M.J.S., and S.H.S. contributed to both the draft and final versions of the manuscript. Contributed to study design/conduct/analysis: B.Y.G.H., B.H., M.B., S.N., M.S., A.G., K.G.H., M.J.S., and S.H.S. 
Data availability The datasets used and/or analyzed during the current study are available from the corresponding author on reasonable request.

\section{Declarations}

Ethics approval and consent to participate Ethics Committee of Babol University of Medical Sciences, Babol, Iran (code IR.MUBABOL.HRI. REC.1399.344).

Consent for publication Yes.

Disclosures None.

\section{References}

1. Shah K, Saxena D, Mavalankar D (2020) Secondary attack rate of COVID-19 in household contacts: a systematic review. QJM 113(12):841-850. https://doi.org/10.1093/qjmed/hcaa232

2. Tobaiqy M, Qashqary M, Al-Dahery S, Mujallad A, Hershan AA, Kamal MA, Helmi N (2020) Therapeutic management of patients with COVID-19: a systematic review. Infection Prevention in Practice 2(3):100061. https://doi.org/10.1016/j.infpip. 2020.100061

3. Gentile I, Maraolo AE, Piscitelli P, Colao A (2020) COVID-19: time for post-exposure prophylaxis? Int J Environ Res Public Health 17(11):3997. https://doi.org/10.3390/ijerph17113997

4. Ip A, Ahn J, Zhou Y, et al. Hydroxychloroquine in the treatment of outpatients with mildly symptomatic COVID-19: a multi-center observational study. MedRxiv. 2020. https://doi.org/10.1101/ 2020.08.20.20178772

5. Peiris M, Leung GM (2020) What can we expect from first-generation COVID-19 vaccines? Lancet 396(10261):1467-1469. https://doi.org/10.1016/S0140-6736(20)31976-0

6. Cortegiani A, Ingoglia G, Ippolito M, Giarratano A, Einav S (2020) A systematic review on the efficacy and safety of chloroquine for the treatment of COVID-19. J Crit Care 57:279-283. https://doi.org/10.1016/j.jcrc.2020.03.005

7. Emami A, Javanmardi F, Pirbonyeh N, Akbari A (2020) Prevalence of underlying diseases in hospitalized patients with COVID19: a systematic review and meta-analysis. Arch Acad Emerg Med 8(1):e35

8. Zhong J, Shen G, Yang H et al (2020) COVID-19 in patients with rheumatic disease in Hubei province, China: a multicentre retrospective observational study. Lancet Rheumatol 2(9):e557-e564. https://doi.org/10.1016/S2665-9913(20)30227-7

9. Bozzalla Cassione E, Zanframundo G, Biglia A, Codullo V, Montecucco C, Cavagna L (2020) COVID-19 infection in a northernItalian cohort of systemic lupus erythematosus assessed by telemedicine. Ann Rheum Dis 79(10):1382-1383. https://doi.org/ 10.1136/annrheumdis-2020-217717

10. Meroni PL, Zavaglia D, Girmenia C (2018) Vaccinations in adults with rheumatoid arthritis in an era of new disease-modifying antirheumatic drugs. Clin Exp Rheumatol 36:317-328

11. Caporali R, Caprioli M, Bobbio-Pallavicini F, Montecucco C (2008) DMARDS and infections in rheumatoid arthritis. Autoimmun Rev 8(2):139-143. https://doi.org/10.1016/j.aut

12. Gianfrancesco M, Hyrich KL, Al-Adely S, Carmona L, Danila MI, Gossec L et al (2020) Characteristics associated with hospitalisation for COVID-19 in people with rheumatic disease: data from the COVID-19 Global Rheumatology Alliance physician-reported registry. Ann Rheum Dis 79(7):859-866

13. Schoot TS, Kerckhoffs APM, Hilbrands LB, van Marum RJ (2020) Immunosuppressive drugs and COVID-19: a review. Front Pharmacol 28(11):1333. https://doi.org/10.3389/fphar.2020.01333

14. Figueroa-Parra G, Aguirre-Garcia GM, Gamboa-Alonso CM, Camacho-Ortiz A, Galarza-Delgado DA (2020) Are my patients with rheumatic diseases at higher risk of COVID-19? Ann Rheum Dis 79(6):839-840. https://doi.org/10.1136/annrh eumdis-2020-217322

15. Ferreira A, Oliveira-e-Silva A, Bettencourt P (2020) Chronic treatment with hydroxychloroquine and SARS-CoV-2 infection. MedRxiv. https://doi.org/10.1101/2020.06.26.20056507

16. European Centre for Disease Prevention and Control. Case definition for coronavirus disease 2019 (COVID-19), as of 29 May 2020.https://www.ecdc.europa.eu/en/case-definition-andeu ropean-surveillance-human-infection-novel-coronavirus-2019ncov (accessed May 29, 2020)

17. Poustchi H, Darvishian M, Mohammadi Z, Shayanrad A, Delavari, Bahadori monfared A et al. A SARS-CoV-2 antibody seroprevalence in the general population and high-risk occupational groups across 18 cities in Iran: a population-based cross-sectional study. Lancet Infect Dis 200

18. Chih-ChengLaia et al (2020) Population-based seroprevalence surveys of anti-SARS-CoV-2 antibody: an up-to-date review International. J Infect Dis 101:314-332

19. Sarzi-Puttini P, Marotto D, Caporali R, Montecucco CM, Favalli EG, Franceschini F, et al. Prevalence of COVID infections in a population of rheumatic patients from Lombardy and Marche treated with biological drugs or small molecules: a multicentre retrospective study. J Autoimmun. 2020 Sep

20. Hu K, Wang M, Zhao Y, Zhang Y, Wang T, Zheng Z, et al. A small-scale medication of leflunomide as a treatment of COVID19 in an open-label blank-controlled clinical trial.Virol Sin. 2020 Jul 21:1-9. https://doi.org/10.1007/s12250-020-00258-7. Epub ahead of print. PMID: 32696396; PMCID: PMC7371831

21. Wang M, Zhao Y, Hu W, Zhao D, Zhang Y, Wang T et al. Treatment of COVID-19 patients with prolonged post-symptomatic viral shedding with leflunomide - a single-center, randomized, controlled clinical trial. Clin Infect Dis. 2020 Sep 21:ciaa1417. https://doi.org/10.1093/cid/ciaa1417. Epub ahead of print. PMID: 32955081 ; PMCID: PMC7543328.

22. Rajasingham R, Bangdiwala AS, Nicol MR, et al. Hydroxychloroquine as pre-exposure prophylaxis for COVID-19 in healthcare workers: a randomized trial. Preprint. medRxiv. 2020;2020.09.18.20197327. Published 2020 Sep 18. https://doi. org/10.1101/2020.09.18.20197327

23. Abella BS, Jolkovsky EL, Biney BT, Uspal JE, Hyman MC, Frank I, et al. Efficacy and safety of hydroxychloroquine vs placebo for pre-exposure SARS-CoV-2 prophylaxis among health care workers: a randomized clinical trial. JAMA Intern Med. 2020 Sep 30:e206319. https://doi.org/10.1001/jamainternmed.2020.6319. Epub ahead of print. PMID: 33001138; PMCID: PMC7527945

24. Berta Grau-Pujol, Daniel Camprubí, Helena Marti-Soler, Marc Fernández-Pardos, Clara Carreras-Abad, Maria Velasco de Andrés, ElisabetFerrer, Magdalena Muelas-Fernandez, Sophie Jullie et al. Pre-exposure prophylaxis with hydroxychloroquine for COVID-19: initial results of a double-blind, placebo-controlled randomized clinical trial

25. Rentsch CT, DeVito NJ, MacKenna B, Morton CE, Bhaskaran K, Brown JP et al (2020) Effect of pre-exposure use of hydroxychloroquine on COVID-19 mortality: a population-based cohort study in patients with rheumatoid arthritis or systemic lupus erythematosus using the OpenSAFELY platform. Lancet Rheumatol 3(1):e19-27. https://doi.org/10.1016/S2665-9913(20)30378-7 
26. Gentry CA, Humphrey MB, Thind SK, Hendrickson SC, Kurdgelashvili G, Williams RJ II (2020) Long-term hydroxychloroquine use in patients with rheumatic conditions and development of SARS-CoV-2 infection: a retrospective cohort study. Lancet Rheumatol. https://doi.org/10.1016/S2665-9913(20)3030

27. Boulware DR, Pullen MF, Bangdiwala AS, Pastick KA, Lofgren SM, Okafor EC et al (2020) A Randomized trial of hydroxychloroquine as postexposure prophylaxis for Covid-19. N Engl J Med 383(6):517-525. https://doi.org/10.1056/NEJMoa2016638

28. Cohen MS. Hydroxychloroquine for the prevention of COVID-19 — searching for evidence. N Engl J Med. 2020. https://doi.org/10. 1056/NEJMe20203885

29. Meo SA, Klonoff DC, Akram J (2020) Efficacy of chloroquine and hydroxychloroquine in the treatment of COVID-19. Eur Rev Med Pharmacol Sci 24(8):4539-4547. https://doi.org/10.26355/ eurrev_202004_21038

30. Mudit Agarwal, Piyush Ranjan, Ankit Mittal \& Upendra Baitha (2020): Use of hydroxychloroquine for pre-exposure prophylaxis in COVID 19: debate and suggested future course, Expert Review of Anti-infective Therapy, https://doi.org/10.1080/14787210. 2021.1828062

31. Bhattacharya R, Chowdhury S, Mukherjee R et al (2020) Pre exposure hydroxychloroquine use is associated with reduced
COVID19 risk in healthcare workers. MedRxiv. https://doi.org/ 10.1101/2020,06(09),pp.20116806

32. Chatterjee P, Anand T, Singh KJ, Rasaily R, Singh R, Das S et al (2020) Healthcare workers \& SARS-CoV-2 infection in India: a case-control investigation in the time of COVID-19. Indian J Med Res 151(5):459-467. https://doi.org/10.4103/ijmr.IJMR_2234_20

33. Balevic SJ, Hornik CP, Green TP, Clowse MEB, Gonzalez D, Maharaj AR, et al . Hydroxychloroquine in patients with rheumatic disease complicated by COVID-19: clarifying target exposures and the need for clinical trials. J Rheumatol. 2020 May 11:jrheum.200493. https://doi.org/10.3899/jrheum. 200493. Epub ahead of print. PMID: 32393664; PMCID: PMC7655510

34 Mathur G, Mathur S (2020) Antibody testing for COVID-19. Am J Clin Pathol 154(1):1-3. https://doi.org/10.1093/ajcp/aqaa082

35. Castro R, Luz PM, Wakimoto MD, Veloso VG, Grinsztejn B, Perazzo H (2020) COVID-19: a meta-analysis of diagnostic test accuracy of commercial assays registered in Brazil. Braz J Infect Dis 24(2):180-187. https://doi.org/10.1016/j.bjid.2020. 04.003

Publisher's note Springer Nature remains neutral with regard to jurisdictional claims in published maps and institutional affiliations. 